\title{
La Influencia de la Gestión del Conocimiento en la Gestión del Riesgo de Desastres en el Perú
}

\author{
The Influence of Knowledge Management in Peruvian \\ Disaster Risk
}

\begin{abstract}
RESUMEN
El presente artículo fundamenta cómo la Teoría de la Gestión del Conocimiento puede influir en la Gestión del Riesgo de Desastres en el Perú; asimismo, reconoce la importancia de su aplicación con la finalidad de contribuir a incrementar la eficiencia en los procesos de Estimación, Prevención y Respuesta. La Gestión del Conocimiento es una herramienta útil para gestionar, adquirir, analizar, almacenar y compartir conocimiento para resolver los problemas de la Gestión del Riesgo de Desastres en el Perú; al mismo tiempo proporciona al Sistema Nacional de Gestión del Riesgo de Desastres una plataforma común para la coordinación y colaboración interinstitucional, el empleo y uso del conocimiento como fin ulterior permitirá contribuir con la protección de la persona humana y la sociedad.
\end{abstract}

Palabras claves: Gestión del Conocimiento; Gestión del Riesgo de Desastres y Modelo de Gestión del Conocimiento.

\begin{abstract}
This article is based as the Theory of Knowledge Management can influence Disaster Risk Management in Peru; recognizes the importance of your application with the aim of contributing to increase the efficiency in the processes of Estimation of the Risk, Prevention and Response to the Disasters. Knowledge Management is a useful tool for managing, acquiring, analyzing, storing and sharing knowledge to solve the problems of Disaster Risk Management in Peru; at the same time, it provides the National Disaster Risk Management System with a common platform for inter-agency coordination and collaboration, the employment and use of knowledge as a further purpose to contribute to contribute to the protection of the human person and society.
\end{abstract}

Keywords: Knowledge Management; Disaster Risk Management and Knowledge Management Model.

Presentado: 22/11/2019 - Aceptado: 17/12/2019 - Publicado: 20/10/2020

(C) Los autores. Este artículo es publicado por Gestión en el Tercer Milenio de la Facultad de Ciencias Administrativas de la Universidad Nacional Mayor de San Marcos. Este es un artículo de acceso abierto, distribuido bajo los términos de la licencia Creative Commons Atribucion - No Comercia_Compartir Igual 4.0 Internacional. (http://creativecommons.org/licenses/by-nc-sa/4.0/) que permite el uso no comercial, distribución y reproducción en cualquier medio, siempre que la obra original sea debidamente citada. 


\section{INTRODUCCIÓN}

El objetivo de esta investigación es determinar cómo la Gestión del Conocimiento puede influir en la eficiencia de la Gestión del Riesgo de Desastres en el Perú.

La Teoría de la Gestión del Conocimiento como variable independiente nos proporciona la base teórica del presente estudio, existen muchas definiciones sobre el concepto de Gestión del Conocimiento. Las definiciones tratan de sintetizar qué persigue, el porqué de su importancia, cómo se emplea y la necesidad de aplicarla, su influencia es innegable en la actual y denominada sociedad del conocimiento; autores y organizaciones le dan su propio significado pero con muchas coincidencias. Podemos citar algunas definiciones:

Para Nonaka y Takeuchi (1995) en la organización creadora del conocimiento afirman que es necesario "(...) que la capacidad de una empresa en su conjunto para crear nuevos conocimientos, así como difundirlos en toda la organización y que queden establecidos en productos, servicios y sistemas" (p. 3).

El autor establece una teoría sobre la creación del conocimiento argumentando que el conocimiento es inicialmente creado por los individuos y se convierte en conocimiento organizacional mediante un proceso o modelo establecido, refiriendo que:

(...) necesitamos una nueva teoría de la creación del conocimiento organizacional, tendrá su propia epistemología (...) cuya piedra angular es la distinción entre el conocimiento tácito y explícito, la clave de la creación del conocimiento es la movilización y conversión del conocimiento tácito, en cuanto a la dimensión ontológica se centra en los niveles de las entidades creadoras del conocimiento (individual, grupal, organizacional e inter organizacional). (Nonaka y Takeuchi, 1995, p. 62)

Otros autores como Hernández (2016) afirma que "es el proceso de captar, desarrollar, compartir y utilizar efectivamente el conocimiento organizativo, con un enfoque multidisciplinario, orientado al logro de los objetivos organizacionales, haciendo el mejor uso del conocimiento" (p. 9).
La definición propuesta por Del Moral, Pazos, Rodriguez, Rodriguez-Paton y Suarez (2007) es:

El conjunto de principios, métodos, técnicas herramientas, métricas y tecnologías que permiten obtener conocimientos precisos, para quienes lo necesitan, del modo adecuado, en el tiempo oportuno de la forma más eficiente y sencilla, con el fin de conseguir una actuación institucional lo más inteligentemente posible. (pp. 18-19)

La definición de Osorio (2003) es:

El conjunto de procesos o sistemas que permiten que el capital intelectual de una organización aumente en forma significativa, mediante la gestión de capacidades de resolución de problemas de forma eficiente y en el menor tiempo posible con el objetivo final de generar ventajas competitivas sostenibles en el tiempo.

Diversas organizaciones tienen significativas descripciones e interpretaciones sobre la Gestión del conocimiento, como se cita en Del Moral consolidando algunas de las más importantes.

Para Macintosh, Filby y Tate (1998) implica:

La identificación y análisis del conocimiento requerido y disponible y la subsiguiente planificación y control de las acciones para desarrollar activos de conocimientos para alcanzar los objetivos de la institución.

Para Maglitta (1995) la Gestión del Conocimiento es:

Organizar y hacer disponible algo tan importante como saber cómo, dónde y cuándo. Incluye procesos, procedimientos, patentes, referencias a trabajos, fórmulas, mejores prácticas, previsiones y elecciones de tecnologías como Intranets. Groupware, almacenes de datos, redes, tablones de boletines de videoconferencias, todas son herramientas claves para almacenar y distribuir esta inteligencia.

Para O’Dell (1996) significa la "aplicación de enfoques sistemáticos para encontrar, entender y usar conocimientos para crear valor".

Para Hibbard (1997) es: 
Proceso de capturar la experiencia de una institución en cualquier parte donde resida. Conservarla en bases de datos, en papel o en la mente de las personas y distribuirla a cualquier sitio donde pueda ayudar a dar la más alta rentabilidad. (p. 46)

Para Del Moral, Pazos, Rodriguez, Rodriguez-Paton y Suarez (2007) es:

Organizar y analizar información en bases de datos de una institución para que el conocimiento pueda ser fácilmente compartido en la institución, en lugar de languidecer en el departamento en el que fue creado, inaccesible a los miembros de la institución.

Respecto a nuestra variable dependiente la Política Nacional de Gestión del Riesgo de Desastres, se fundamenta en el seguimiento y control del origen de las amenazas o fenómenos peligrosos y las condiciones de vulnerabilidad de la sociedad, su análisis determina el riesgo asociado para establecer escenarios con la finalidad de evitar su generación, reducirlos o programar una respuesta eficaz ante un desastre inminente.

La Organización de las Naciones Unidas (ONU) en el Marco de Acción de Hyogo para el 2005-2015 aprobó el 22 de enero de 2005 en la novena sesión plenaria, el instrumento más importante para la implementación de la reducción del riesgo de desastres, instrumento que adoptaron los estados miembros de las Naciones Unidas, teniendo como objetivo general aumentar la resiliencia de las naciones y las comunidades ante los desastres. (ONU, 2005)

Este instrumento constituye el inicio del cambio de enfoque a nivel mundial, pasar de una actitud reactiva ante los desastres a una manera diferente de enfrentarlos, una filosofía prospectiva basada en el registro de información y en el control del riesgo, es decir un enfoque estratégico y sistemático para reducir la vulnerabilidad ante las amenazas.

El enfoque prospectivo y reactivo propuesto por el marco de acción de Hyogo marcó la pauta a las naciones del mundo en cuanto a la reducción de la vulnerabilidad y al control de las amenazas en los desastres; como un componente indispensable en todo proceso de desarrollo sostenible, razón por la cual la Presidencia del Consejo de Ministros (PCM) del estado Peruano considera que:

La Gestión del Riesgo de Desastres en el Perú no solo debe tener presencia en el Acuerdo Nacional, sino que debe tener identidad propia y ser definida como una Política Nacional, pues dada su connotación y el nivel de compromiso que demanda debe ser lograda y tratada de modo sistémico y programático. Se basa en la investigación científica y en el registro de informaciones; y orienta las políticas, estrategias y acciones en todos los niveles de gobierno y de la sociedad con la finalidad de proteger la vida de la población y el patrimonio de las personas y del Estado, para contribuir al desarrollo sostenible del país. (PCM, 2012, p. 03).

La base para la Gestión del Conocimiento en la Gestión del Riesgo de Desastres es el conocimiento de las amenazas y las vulnerabilidades que al conjugarse en el tiempo y el espacio generan los riesgos, entendiendo al riesgo como la probabilidad de ocurrencia de un impacto negativo, que al concretarse genera el desastre.

Las amenazas son los factores externos que pueden dañar y las vulnerabilidades son las condiciones de la sociedad susceptibles a ser afectadas por las amenazas o los denominados peligros. Cuando una de estas dos variables no converge, no hay probabilidad del desastre.

Es necesario que el Estado genere un mayor conocimiento sobre las amenazas o peligros, conocer las causas de su origen, los fenómenos físicos pueden ser de origen natural o causados por el hombre y son potencialmente dañinos, estos eventos se presentan en un lugar específico, con una cierta intensidad y en un periodo de tiempo y frecuencia definida.

La tipología de las amenazas como: inundaciones, sismos, deslizamientos sequías y heladas, son factores externos que dañan y hasta cierto punto se pueden analizar, conocer, caracterizar o modelar, pero no controlar como las vulnerabilidades. 
La vulnerabilidad está relacionada con la susceptibilidad de la población a la estructura física o actividad socioeconómica de sufrir daño por acción de las amenazas (PCM, 2011). Las causas de vulnerabilidades son variadas entre las principales está el uso y la ocupación inadecuada del suelo, el aumento de la población, incumplimiento de normas urbanísticas y de construcción y se caracterizan para su estudio en tipos como: física, social, ambiental y económica, el estado requiere conocerlas, evaluarlas, conocer donde están concentradas las poblaciones que requieren de mayor atención ante la exposición de los peligros.

Es necesario conocer y evaluar el acceso básico a los servicios, la densidad poblacional, los niveles de pobreza, de desnutrición, de alfabetismo para determinar su fragilidad ante una exposición de la amenaza, así como evaluar la resistencia de la infraestructura física.

\section{MÉTODOS}

La metodología que se empleó para determinar si la Gestión del Conocimiento influye en la eficiencia de la Gestión del Riesgo de Desastres (GDR) fue el análisis de cuestionarios aplicados a las personas de diferentes organismos públicos a cargo de la GRD, principalmente las que cumplen labores del manejo de la información, como Organismos Ejecutores y Ministerios, personas destacadas al Centro de Operaciones de Emergencia Nacional (COEN), área física implementada por el Instituto Nacional de Defensa Civil (INDECI) junto a demás componentes del Sistema Nacional de Gestión del Riesgo de Desastres, para reunir, monitorear y analizar la información sobre riesgos.

\section{RESULTADOS}

Se analizaron los factores en donde la Gestión del conocimiento puede contribuir con: el planeamiento, articulación y el financiamiento público, estructura de las organizaciones, determinación de infraestructura y equipamiento logístico, determinación de la política, gestión de recursos humanos, evaluación de resultados, seguimiento y monitoreo y gestión por procesos. De acuerdo a la Tabla 1, se puede apreciar que el $7.27 \%$ del personal cuestionado respondió que definitivamente la Gestión del Conocimiento no debería influir en los factores identificados como problemática de la Gestión del Riesgo de Desastres en el Perú, el 11.6\% considera que probablemente no influye, el $5 \%$ se muestra indeciso sin embargo el $26.3 \%$ considera que probablemente sí influye y el $49.6 \%$ afirma que definitivamente sí. En síntesis, quiere decir que definitivamente la Gestión del Conocimiento es una herramienta útil y que influye en los factores identificados como problemática y que afectan directamente la Gestión del Riesgo de Desastres.

\section{DISCUSIÓN}

Consideré para interés de nuestra investigación tener como base la teoría de la creación del conocimiento organizacional, desarrollada por Nonaka y Takeuchi (1995), quien explica el fenómeno de la creación del conocimiento organizacional de una forma verdadera y justificada desde el punto de vista de la capacidad de las organizaciones para crear nuevos conocimientos, difundirlos en toda la organización a manera de un espiral y que estos queden establecidos en los productos, servicios y sistemas.

Tabla 1.

La Gestión del Conocimiento influye en la problemática de la Gestión del Riesgo de Desastres en el Perú.

\begin{tabular}{lcccc} 
Categorías & Frecuencia & Porcentaje & $\begin{array}{c}\text { Porcentaje } \\
\text { válido }\end{array}$ & $\begin{array}{c}\text { Porcentaje } \\
\text { acumulado }\end{array}$ \\
\hline Definitivamente no & 70 & 7.27 & 7.27 & 7.27 \\
Probablemente no & 112 & 11.63 & 11.63 & 18.90 \\
Indeciso & 49 & 5.09 & 5.09 & 23.99 \\
Probablemente si & 254 & 26.38 & 26.38 & 50.36 \\
Definitivamente si & 478 & 49.64 & 49.64 & $\mathbf{1 0 0 . 0 0}$ \\
Total & $\mathbf{9 6 3}$ & $\mathbf{1 0 0 \%}$ & $\mathbf{1 0 0} \%$ & \\
\hline
\end{tabular}

Fuente. Elaboración propia 
Como citan los autores, el conocimiento en un inicio es creado por los individuos dentro de las organizaciones y la gestión consiste en convertir este en un conocimiento organizacional, mediante un proceso establecido por la teoría, desde una dimensión epistemológica es decir que "la clave de la creación del conocimiento es la movilización y conversión del conocimiento tácito" (Nonaka y Takeuchi, 1995, p. 31). Por otro lado la teoría muestra también una dimensión ontológica centrada en los niveles de las entidades creadoras del conocimiento (individual, grupal organizacional e interinstitucional), es en este contexto que el autor en un plano cartesiano de dos ejes (epistemológico y ontológico) identifica el crecimiento espiral del conocimiento y como este se crea en su interacción:

Cuando la interacción entre conocimiento tácito y explícito se eleva dinámicamente de un nivel ontológico bajo a niveles más altos surge un espiral, la parte central de la teoría es cómo se da esa espiral, determinando las cuatro formas de conversión del conocimiento que surgen cuando el conocimiento tácito y el explícito interactúan. (Nonaka y Takeuchi, 1995, p.62) (Ver Figura 1).
Antes de describir las cuatro formas de la creación del conocimiento, en este punto es conveniente reflexionar sobre nuestra variable, Gestión del Riesgo de Desastres, e identificar la interacción de los actores en el modelo propuesto por Nonaka (1995), desde el punto de vista epistemológico podemos determinar que los individuos, las unidades sociales, localidades y regiones son expuestas a las amenazas, en condiciones de vulnerabilidad, el individuo y sus unidades sociales es el origen y el fin de la generación de conocimiento, es donde el conocimiento tácito individual se origina, las entidades públicas, en todos los niveles del gobierno (Nacional, Regional y Local) interactúan para explicitar, materializar y hacer evidente el conocimiento y determinar medidas estructurales y no estructurales para "la prevención, la reducción y el control permanente de los factores de riesgo de desastres en la sociedad"; conocemos que la gestión del riesgo es un procesos social donde el fin y el objeto es el ciudadano habitante junto con las unidades sociales que conforma, el aspecto ontológico de la teoría propuesta por Nonaka y Takeuchi (1995), nos invita a reconocer dentro de esta dimensión a todos los actores del Sistema Nacional de la Gestión del Riesgo en el Perú, que de alguna manera interactúan para establecer el espiral como un "Sistema interinstitucional,

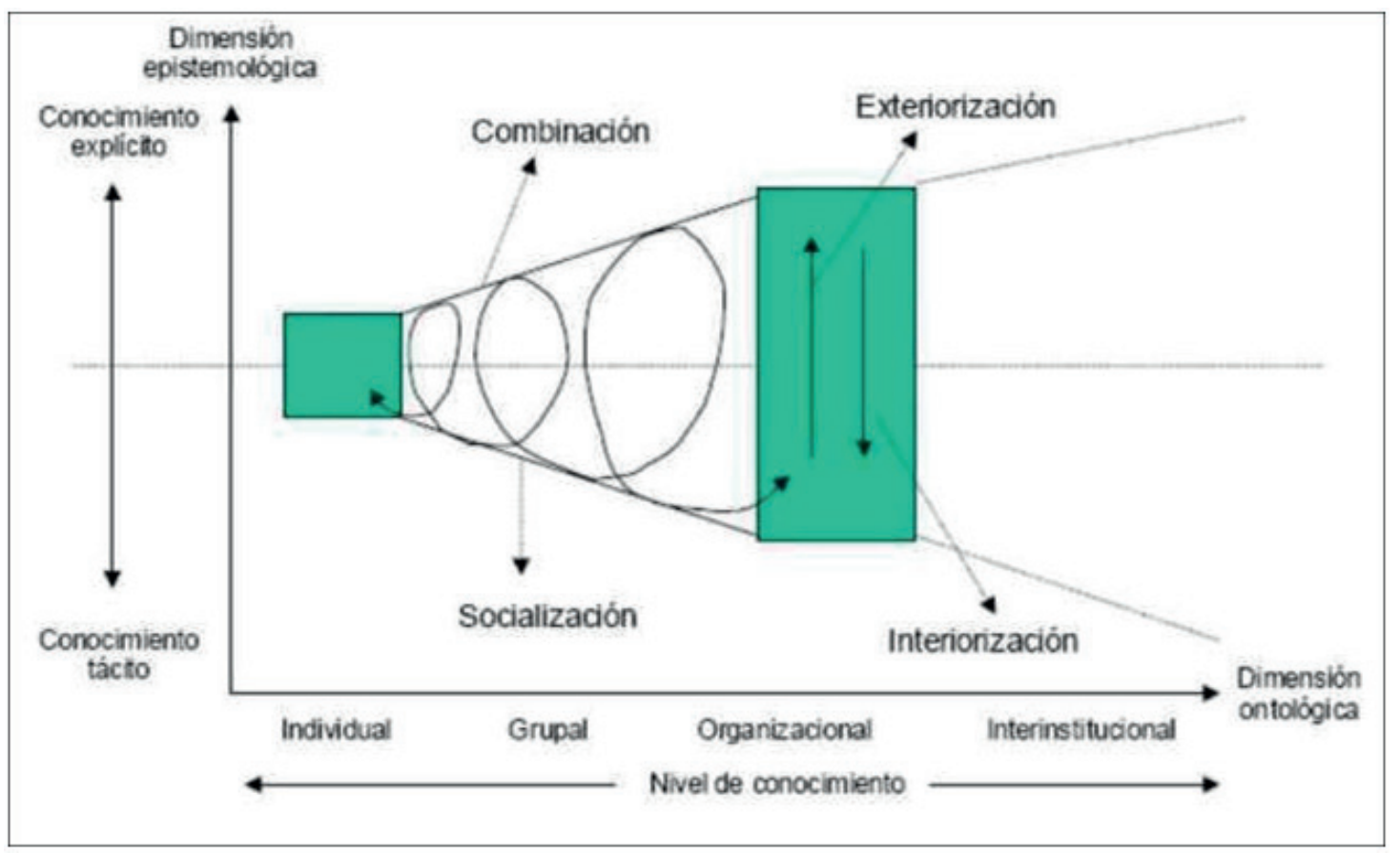

Figura 1. Espiral del conocimiento organizacional. Adaptado de "The Knowledge Creating Company" por Nonoka \& Takeuchi, 1995. 
sinérgico, descentralizado, transversal y participativo con la finalidad de identificar y reducir los riesgos asociados a los peligros o minimizar sus efectos".

Desde la Presidencia del Consejo de Ministros en su rol articulador e integrador del Nivel Gobierno Nacional y sus ministerios (Economía, Defensa, Salud, Educación, Interior, Ambiente, Agricultura, Transportes y Vivienda) a los organismos ejecutores como el Centro Nacional de Estimación, Prevención y Reducción del Riesgo de Desastres (CENEPRED), para conducir los procesos de estimación, prevención y reducción; y el INDECI, que integra las acciones de preparación y respuesta, para el manejo de los desastres. Creemos necesario también, identificar las unidades sociales, como un nivel individual; las localidades y regiones, como el nivel grupal; los organismos ejecutores y entidades públicas nacionales, como nivel organizacional; la Presidencia del Consejo de Ministros ente rector del Sistema Nacional de Gestión del Riesgo de Desastres (SINAGERD), como el nivel interinstitucional.

Respondiendo a la pregunta ¿cómo generar conocimiento en el Sistema Nacional de la Gestión del Riesgo de Desastres?, nuevamente nos apoyaremos en Nonaka (1995) para determinar cómo su método para la conversión del conocimiento, se explica mediante la interacción del conocimiento tácito y explícito; desde el punto de vista de los autores estas dos entidades no son separadas sino complementarias.

Hay una interacción e intercambio entre el conocimiento tácito e implícito en las actividades creativas de los seres humanos (...). Nuestro modelo dinámico de la creación del conocimiento se fundamenta en el supuesto crítico de que el conocimiento humano se crea y se expande a través de la interacción social del conocimiento tácito y conocimiento explícito. A esta interacciona la llamamos conversión del conocimiento. (Nonaka y Takeuchi, 1995, p. 57)

Luego de haber analizado los aspectos etimológicos sobre el conocimiento, revisar las precisiones del autor sobre el conocimiento e información y para nuestro caso definido los actores ontológicos en el Sistema Nacional de Gestión del Riesgo de Desastres corresponden en esta parte reflexionar sobre cómo la teoría de Nonaka (1995) explica el fenómeno de la creación del conocimiento, describiendo cuatro formas de conversión del conocimiento, (ver Figura 2).

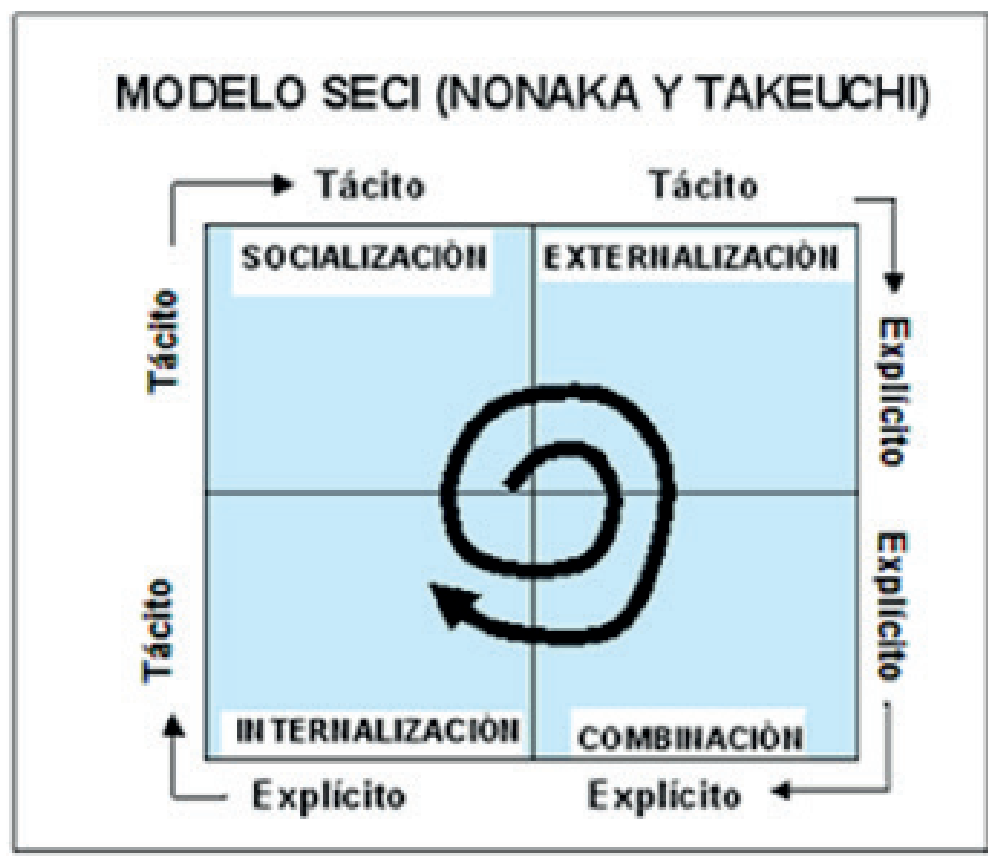

Figura 2. Modos para la creación del conocimiento. Adaptado de "The Knowledge Creating Company" por Nonoka \& Takeuchi, 1995, p. 81. 
Esta teoría permite reflexionar sobre las posibles formas que el Sistema Nacional de Gestión del Riesgo tiene para identificar las fuentes de conocimiento en cada uno de los procesos de nuestra investigación (Estimación del Riesgo, Prevención, Reducción y Manejo de Desastres). Los autores del modelo afirman que:

Asumir que el conocimiento se crea por la interacción entre el conocimiento tácito a explícito nos permite postular cuatro formas de conversión de conocimiento: 1 . de tácito a tácito, que llamamos socialización; 2. de tácito a explícito, o exteriorización; 3. De explícito a explícito, o combinación y 4. de explícito a tácito o interiorización. (Nonaka y Takeuchi, 1995, p. 69)

La Socialización (Tácito a Tácito), según Nonaka y Takeuchi (1995) es "un proceso que consiste en compartir experiencias y, por tanto crear conocimiento tácito tal como los modelos mentales compartidos y las habilidades técnicas" (p. 70); es un proceso de interacción entre los individuos mediante la observación, imitación y práctica, la clave es la adquisición del conocimiento mediante la experiencia Nonaka (1995) afirma que "sin alguna forma de experiencia compartida, a una persona le resulta extremadamente difícil proyectarse a sí misma al interior del pensamiento de otra persona" (p. 70).

La transferencia de la información carecerá de sentido en la Gestión del Riesgo de Desastres mientras no se transfieran las emociones referidas a la vulnerabilidad y a la capacidad de las amenazas y a su contexto específico en cada una de las unidades sociales, un ejemplo de sociabilización para la gestión del riesgo podrían ser las tormentas de ideas, reuniones informales para llevar a cabo discusiones detalladas y resolver los problemas que produce el riesgo, mediante la participación comunitaria para compartir las experiencias y fomentar la confianza en la aplicación de las acciones de reducción y prevención; el proceso de sociabilización a través de la observación y la práctica podría convertirse en un proceso cíclico de intercambio de conocimiento tácito y de creación de ideas para mejorar las condiciones de vulnerabilidad.

Exteriorización (de tácito a explícito), para Nonaka y Takeuchi (1995) consiste en:
Un proceso a través del cual se enuncia el conocimiento tácito en forma de conceptos explícitos (...), y adopta la forma de metáforas, conceptos, hipótesis o modelos (...). Escribir es un acto de conversión de conocimiento tácito en conocimiento enunciable; (...). Promueven la reflexión y la interacción entre los individuos. La exteriorización se observa típicamente en el proceso de creación de conceptos y es generada por el diálogo o la reflexión colectiva. (p. 72)

En esta forma de adquisición se hace necesario la interacción entre las instituciones públicas, los organismos públicos y los organismos ejecutores de la GRD con los individuos y las unidades sociales para el intercambio de conocimiento a través de la reconfiguración de la información, contextualización, re-categorización de conocimientos explícitos para conducir a nuevos conocimientos, con el apoyo de las tecnologías de información mediante el empleo de inventarios sistemáticos de desastres y pérdidas, monitoreo de amenazas y pronósticos, evaluación de riesgos, con esta información exteriorizamos los conceptos explícitos como afirman los autores:

una vez que son creados, los conceptos explícitos pueden traducirse en un modelo. En un modelo lógico no debe de haber contradicciones y todos los conceptos y proposiciones deben de expresarse usando un lenguaje sistemático y una lógica coherente. (Nonaka y Takeuchi, 1995, p. 76)

Combinación (de explícito a explícito); según los autores se trata de un proceso de sistematización de conceptos, buscan captar la idea mediante la combinación de conceptos y análisis y su intercambio entre todos los componentes del sistema, los autores consideran que este intercambio se puede dar de la siguiente manera: "mediante medios, tales como documentos, juntas, reconfiguración de la información existente, clasificando, añadiendo, codificando en bases de datos, la educación y en entrenamiento formal" (Nonaka y Takeuchi, 1995, p. 77).

Este proceso de interacción mutua hace necesario la integración del Sistema Nacional 
mediante el empleo del Sistema Nacional de Información para la Gestión del Riesgo de Desastres, bajo el liderazgo de la Presidencia del Consejo de Ministros se requiere tener una basada en información científica y del registro de informaciones para orientar las políticas y estrategias y acciones en todos los niveles ontológicos identificados.

Por último, la Interiorización (de explícito a explícito), esta forma está relacionada según los autores con el "Aprendiendo haciendo"; sin embargo para que el conocimiento explícito se vuelva tácito, es de gran ayuda que el conocimiento se verbalice o diagrame en documentos. La documentación ayuda a los individuos lo que se ha experimentado enriqueciéndolo, empezando así una nueva espiral de creación del conocimiento. (Nonaka y Takeuchi, 1995, pp. 78-79)

Sobre esta forma de creación podemos reafirmar que el conocimiento es requerido para aplicarlo y transformarlo en acciones estructurales y no estructurales, una forma de documentar las acciones es el seguimiento de los planes para las acciones de reducción y prevención, por ejemplo la implementación del uso adecuado del suelo y la planificación urbana, mejoramiento de viviendas, intervención de cuencas, aplicación de las normas de construcción, implementación de técnicas para fenómenos peligrosos, planeamiento de las acciones de respuesta para el manejo de desastres.

\section{CONCLUSIONES}

Se determinó que la Gestión del Conocimiento influye en los factores identificados como problemática de la Gestión del Riesgo de Desastres en el Perú, esto quiere decir que incide en la eficiencia del planeamiento, articulación del financiamiento público, determinación de la infraestructura y equipamiento, política de GRD, gestión de recursos humanos, evaluación de resultados, seguimiento y monitoreo y en la gestión de procesos eficientes para la GRD; en síntesis podemos concluir que la Gestión del Conocimiento es una herramienta útil para resolver los problemas identificados en la Gestión Pública que afectan directamente a la Gestión de Riesgo de Desastres.

\section{REFERENCIAS BIBLIOGRÁFICAS}

Del Moral, A., Pazos, J., Rodríguez, E., Rodríguez-Patón, A. y Suárez, S. (2007). Gestión del Conocimiento. Madrid, España: Thomson.

Hernández, V. (2016). La Gestión del Conocimiento en las organizaciones. Buenos Aires: Alfaomega.

Hibbard, J. (1997). Knowing what we know'. Information Week.

Macintosh, A., Filby, I. y Tate, A. (1998) . Knowledge Asset Road Maps. 2da International Conference on Practical Aspects of Knowledge Management. Switzerland.

Maglitta, J. (1995). Smarten up. ComputerWorld, 84.

Nonaka, I. y Takeuchi, H. (1995). Un Modelo para la Gestión del Conocimiento Organizacional. México: Oxford University Press.

O’Dell, C. (1996). A current review of knowledge management best practice. Conference on Knowledge Management and the Transfer of Best Practices, Business Intelligence, London.

Organización de las Naciones Unidas. (2005). Marco de Acción de Hyogo para el 2005-2015. Aumento de la Resiliencia de las naciones y las comunidades en desastres. Hyogo.

Osorio, M. (2003). El capital intelectual en la gestión del conocimiento. ACIMED, 11(6)

Presidencia del Consejo de Ministros. (2011). Reglamento de la Ley $N^{\circ}$ 29664. Lima: Aprobado con DS № 048. 